\title{
Contextualism, Relativism and the Liar*
}

\author{
Gil Sagi ${ }^{+}$
}

November 4, 2016

\begin{abstract}
Contextualist theories of truth appeal to context to solve the liar paradox: different stages of reasoning occur in different contexts, and so the contradiction is dispelled. The word 'true' is relativized by the contextualists to contexts of use. This paper shows that contextualist approaches to the liar are committed to a form of semantic relativism: that the truth value of some sentences depends on the context of assessment, as well as the context of use. In particular, it is shown how Simmons's and Glanzberg's contextualist approaches entail relativism. In both cases, the liar sentence gets different semantic evaluations as uttered in a fixed context of use but assessed from different contexts. Shift in context of use alone cannot provide the full explanation of the liar. These contextualist approaches, as originally presented, were thus mischaracterised and they should be re-evaluated according to their full implications.
\end{abstract}

Keywords: liar paradox, contextualism, relativism

\section{Introduction}

Contextualist theories of truth appeal to context to explain or dissolve the liar paradox: different stages of reasoning occur in different contexts, and so the contradiction is dispelled. What is meant by contextualism is relativity of semantic value or extension to context. Specifically, it is the context of use or utterance of an expression that

*The final publication is available at Springer via http://link.springer.com/article/10.1007/s10670016-9850-6.

${ }^{\dagger}$ Philosophy Department, University of Haifa, 199 Aba Khoushy Ave, Mt. Carmel, Haifa 3498838, Israel凹gilisagi@gmail.com 
is referred to. Contextualism as a solution to the liar has been endorsed in various forms. ${ }^{1}$ Recently, there have been defenses of another kind of context sensitivity for certain expressions, namely, sensitivity to context of assessment. That is, the context in which a sentence containing the relevant expression is assessed affects the extension, i.e. the truth value of that sentence. This semantic approach is known as relativism. ${ }^{2}$

What happens with the word 'true'? Notice that (generally speaking) when 'true' occurs, some sentence is assessed. So the context of utterance of 'true' is also a context of assessment of some sentence or utterance. This leads to the natural conjecture that contextualism about truth entails relativism about truth. In this paper I give an argument to this effect, and explore further consequences for contextualism and relativism. My aim will not be to defend either view, but rather to show the connection between the views.

In $\S \S 2-3$ I present, in a general way, the contextualist approach to the liar and show that it entails relativism about 'true'. In $\S 4$ I show that relativism about any expression entails both contextualism about 'true' or some other semantic expression, and relativism about 'true', and so we seem to reach a full circle. In §5 I take on two case studies of contextualists about truth, Keith Simmons and Michael Glanzberg, and show how in particular their theories are committed to a form of relativism. In §6 I conclude, leaving open the question of whether the relativist approach entailed by contextualism can serve as a viable account of the liar.

\section{Contextualism and the Strengthened Liar Discourse}

Contextualists appeal to notions from linguistics and the philosophy of language to solve the liar paradox. Thus they employ a natural language perspective on the liar, analyzing the discourse in which a contradiction is seemingly derived. Specifically, they employ the idea of a context shift throughout the discourse, so that sensitivity to context explains conflicting evaluations of the same sentence. I shall leave the notion of context schematic for the sake of generality, so that the argument will apply

\footnotetext{
${ }^{1}$ The earlier examples, to which I will not refer, include: (Parsons, 1974; Burge, 1979; Barwise \& Etchemendy, 1987).

${ }^{2}$ Relativism has been endorsed with respect to, e.g., predicates of taste, epistemic modals and knowledge attributions. In presenting relativism I loosely follow (MacFarlane, 2014). For a variety of relativistic approaches, see (García-Carpintero \& Kölbel, 2008).
} 
most widely. In $\S 3$ I will add some minimal assumptions relating contexts of use and contexts of assessment. What the contextualists I discuss later on do share is that sentences are used in contexts, contexts have a time component, and contexts may be affected by sentences previously uttered. Of course, contexts may (and should) be thought of as richer than that (e.g. as containing also a world and agent component), but here we leave their exact specification open.

The discourse at focus is the one concerning the "strengthened liar", i.e. the sentence that says of itself that it is not true. ${ }^{3}$ The discourse proceeds as follows. ${ }^{4}$ We start out by encountering the (strengthened) liar sentence, labeled (L):

1. (L): $(\mathrm{L})$ is not true.

We then attempt to assign $(\mathrm{L})$ a truth value. On the assumption that $(\mathrm{L})$ is true, $(\mathrm{L})$ then must not be true. On the assumption that $(\mathrm{L})$ is not true, we find that $(\mathrm{L})$ must then be true. (L) resists being evaluated, and thus we infer that:

2. (L) is defective.

Now, since (L) is defective it cannot be true, so we infer:

3. (L) is not true.

But now it seems that we inferred (L) itself, and thus we infer that after all:

4. (L) is true.

We observe that $(\mathrm{L})$ appears twice in this reasoning. After the first time it appears, in line (1), (L) is found to be defective. The second time it appears, in line (3), it is found to be true. This alteration in semantic evaluation is then explained by a context shift: stages (1) and (3) occur in different contexts, and relativizing to contexts there is no contradiction after all.

The primary challenge for contextualists who employ this account of the liar discourse is to explain the feasibility of a context shift between (1) and (3) philosophically

\footnotetext{
${ }^{3}$ The liar here is "strengthened", since by contrast to the ordinary liar that ascribes falsity to itself, the ascription of un-truth resists simple gap-theoretic solutions.

${ }^{4}$ This presentation of the strengthened liar discourse follows (Simmons, 2015) in general lines. I will reformulate the discourse in accordance with the different details in Simmons's and Glanzberg's presentations when I discuss their views.
} 
and formally. Contemporary theories of context provide a useful tool for contextualists about truth. The key idea is that an assertion made in a discourse may change the common background knowledge of the participants and by that change the relevant context (see e.g. (Stalnaker, 1978) and (Lewis, 1979)). Here, in the liar discourse, the information that $(\mathrm{L})$ is defective produces the relevant change. We start out in an unreflective context, where no kind of assessment of the liar is assumed. Realizing that the liar sentence is defective, we are moved to a reflective context in which we restate the liar, and at that time it is true (Simmons, 2015; Glanzberg, 2001; Simmons, 1993; Glanzberg, 2004).

The context at stake, which is taken as an extra parameter in the evaluation of the liar, is the context of use or utterance. We may add the following definition:

- A sentence $s$ is use-sensitive if $s$ 's evaluation depends on the context of use. Specifically, if $s$ is use-sensitive there are contexts $c_{1} \neq c_{2}$ in which $s$ has conflicting semantic values. ${ }^{5}$

The liar sentence is uttered twice, and each time, it seems, it merits a different evaluation. By the above definition, the liar is thus use-sensitive.

At this point, I would like to merely make a note that in each stage of the reasoning, a truth value assessment of some sentence is expressed by use of the object-language truth-predicate. The context of use of that assessment can be viewed as a context of assessment of the sentence mentioned, given that we are willing to countenance assessments in the object language. Thus, (L) is assessed in different stages of the reasoning. Given that there is a context shift somewhere between (1) and (4), (L) is assessed from at least two different contexts. These assessments give different verdicts, and thus it seems that we are prima facie provided with the option of attributing the change in evaluation to the context of assessment rather than the context of use. In the following sections I show that it is the former kind of sensitivity that contextualists are committed to by their own reasoning. There is no conflict between relativism

\footnotetext{
${ }^{5}$ Conflicting values must be distinct. However, not all distinct values are conflicting. I will not define conflicting values, but will rather go case by case. Thus, if $s$ receives 'true' and 'false' in two different contexts of use, $s$ is use-sensitive, but if $s$ receives 'not true' and 'false' in two different contexts of use, $s$ is still not shown to be use-sensitive, since the evaluations are not in conflict. Less obviously, as we shall see, we will need to accept that 'defective' and 'not true' are conflicting values. This is based on the fact that we reached the conclusion that $(\mathrm{L})$ is defective by denying its being not true.
} 
and contextualism in the sense that an expression can be sensitive to both context of assessment and context of use, and the authors I discuss can thus hold both to be true. However, they admit only to holding the latter, and thus they mischaracterize their own approach: shift in context of use does not fully explain the liar reasoning.

\section{From Contextualism to Relativism}

My aim is to show that contextualism about truth, as presented in the previous section, or as endorsed by either Simmons or Glanzberg, entails a form of relativism or assessment sensitivity about truth. I will show that under assumptions that ought to be congenial to the contextualists, 'true' is sensitive to context of assessment. However, if one rejects these assumptions, we are still left with a form of relativity: we shall see that contextualism as presented here at the very least yields relativism in a very general sense, by which truth-value is not determined by a sentence, context of use and circumstance of evaluation.

One crucial assumption that I will make and that will get the stronger argument running is that assessments can be expressed in the object language, so that the various steps in the liar discourse can be treated as assessments of the liar. This assumption might seem foreign to relativists, who ordinarily separate the use of 'true' in the object-language and truth in the metalanguage as a value in the semantic theory (MacFarlane, 2005, p. 315). But the discussion on the liar paradox calls for another perspective, as the goal is to account for an object-language truth predicate in a way that will capture its role as providing a semantic assessment without running into triviality. Indeed, the contextualists we discuss treat the truth predicate as that which expresses the semantic value assigned to the truth bearers, be they sentences or propositions. It is by following the stages of the liar discourse as correctly assigning a semantic value to the liar that the contextualists substantiate their view that there is a context-shift. Thus, given that the general aim of contextualist solutions to the liar is to consistently include 'true' in the object language in a manner faithful to its role in semantic theory, the inclusion of assessments in the object language, or the labeling of truth attributions in the object language as assessments, is a natural move.

An added assumption we make is that contexts of use and contexts of assessment are of the same kind (as in (MacFarlane, 2014, 60)), or are at least correlated so that a 
difference in context of utterance of two assessments of the same utterance amounts to a difference in context of assessment of the utterance assessed. ${ }^{6}$

We note that for the contextualists we are dealing with, the truth bearers are either sentence tokens or propositions. In the latter case, a proposition is determined or expressed by a sentence in context. In what follows I shall refer to such sentence-context pairs as utterances for brevity, and will ignore the possible theoretical differences between utterances, sentence tokens and sentence (types) in context. ${ }^{7}$

We shall make use of the following definitions: Let $\alpha, \beta$ be utterances, $s_{\alpha}$ and $s_{\beta}$ the associated sentences and $c_{\alpha}$ and $c_{\beta}$ the associated contexts of use respectively.

- $\beta$ is an assessment of $\alpha$ if $\beta$ assigns to $\alpha$ (an outcome of) a truth evaluation, e.g. ' $\alpha$ is true', ' $\alpha$ is false', but also ' $\alpha$ is not true', ' $\alpha$ is defective' and ' $\alpha$ has no truth conditions/truth value'. If propositions are taken to be the truth bearers, then assessments will be of the form: ' $\alpha$ expresses a true proposition', ' $\alpha$ expresses a false proposition', as well as ' $\alpha$ does not express a proposition', etc.

Admittedly, this is a vague definition, but since we deal here with clear-cut cases, it will do for our purposes.

- Let $\beta$ be an assessment of $\alpha$. Then $v a l(\alpha, \beta)$ is the value assigned by $\beta$ to $\alpha$. Again, this is a vague notion, but for our purposes it will do.

We define true assessments as a special case of true utterances:

- $\beta$ is a true assessment of $\alpha$ if $\beta$ is an assessment of $\alpha$ and $\beta$ is true with respect to all relevant parameters. ${ }^{8,9}$

\footnotetext{
${ }^{6}$ If those are conceived to be radically different, then we still remain with sensitivity to context of use of an utterance expressing an assessment.

${ }^{7}$ Such differences include utterances being physical speech acts that take time, and sentences-incontext being more abstract theoretical constructs. See (Kaplan, 1989, 546) and (MacFarlane, 2014, 47f).

${ }^{8}$ For our purposes, the relevant parameters are $\beta$ 's given context of use $c_{\beta}$, and some context of assessment. As a default, unless otherwise specified, we shall take the context of assessment of assessments to be equal to their context of use.

${ }^{9}$ The requirement for a true assessment might raise the worry of an infinite regress: from a relativistic standpoint, the truth of an assessment depends on its context of use as well as its own context of assessment. However, there is no need to go further than that, since we do not require a true assessment on the second level, we merely rely on the context in which such an assessment could be made. If relativism
} 
We employ the following principles:

- Principle of contexts of assessment: If $\beta$ is an assessment of $\alpha$, then $\beta$ 's context of use is one of $\alpha$ 's contexts of assessment.

- Principle of true assessments: If $\beta$ is a true assessment of $\alpha$ (relative to a given context of assessment, see f.n. 9), then $\operatorname{val}(\alpha, \beta)$ is a correct semantic value for $\alpha$ as uttered in its context and assessed from $\beta$ 's context.

We are now ready to define the relevant notions of assessment sensitivity:

- An utterance $\alpha$ is assessment-sensitive if $\alpha$ 's evaluation depends on the context of assessment. Specifically, if there are two contexts $c_{1} \neq c_{2}$ and true assessments $\beta$ and $\gamma$ of $\alpha$ such that $c_{1}$ and $c_{2}$ are the respective contexts of use of $\beta$ and $\gamma$, and $\operatorname{val}(\alpha, \beta)$ and $\operatorname{val}(\alpha, \gamma)$ are conflicting, then $\alpha$ is assessment-sensitive. ${ }^{10,11}$

If propositions are involved, then there are two ways in which an utterance can be assessment-sensitive. According to the more common relativistic view, which following MacFarlane we may call truth-value relativism, the truth of propositions depends on contexts of assessments (Egan, Hawthorne, \& Weatherson, 2005; García-Carpintero \& Kölbel, 2008; MacFarlane, 2014). A less common relativistic view, content relativism, relativizes which proposition is expressed by an utterance to a context of assessment (endorsed by (Cappelen, 2008)). In both cases, the truth value assigned derivatively to an utterance will depend on the context of assessment.

- A sentence $s$ is assessment-sensitive if there is an utterance of $s$ that is assessmentsensitive. $^{12}$

required sensitivity to true assessments rather than sensitivity to context of assessment, we would be in danger of a regress. Nonetheless, when determining the truth of an assessment, we shall consider its context of use also as the context of assessment. The assessments we shall deal with, with the exception of the liar, will presumably not be assessment-sensitive.

${ }^{10}$ Again, conflicting values must be distinct but not all distinct values are conflicting. See f.n. 5 .

${ }^{11}$ One may wonder whether for every assessment-sensitive utterance, the conflicting evaluations would find expression in the object language. Here we don't need such a strong assumption for the argument presented to go through: we only need that parts of the liar discourse, which we have analysed as assessments, are expressible in the object language.

${ }^{12}$ Note that it does not suffice that a sentence has conflicting evaluations in different contexts of assessment for it to be assessment-sensitive: the context of use must remain constant, hence, there is an utterance of the sentence that is assessment-sensitive. 
We shall say that a sub-sentential expression is assessment-sensitive if it occurs in assessment-sensitive sentences, and the explanation of their assessment sensitivity hinges on the occurrence of this expression. Thus, we will show the assessment sensitivity of 'true' through the assessment sensitivity of the liar sentence.

My claim is that in the liar scenario, sensitivity to context of use entails assessment sensitivity. Thus, let us grant the contextualist that indeed context has a non-trivial effect in the case of the liar discourse presented in $\S 2$.

For the contextualists, since truth depends on context, the liar should make reference to an utterance rather than just a sentence, and the liar discourse should be reformulated along these lines:

1. $\left(\mathrm{L}_{u}\right):\left(\mathrm{L}_{u}\right)$ is not true.

2. $\left(\mathrm{L}_{u}\right)$ is defective.

3. $\left(\mathrm{L}_{\mathcal{u}^{\prime}}\right):\left(\mathrm{L}_{u}\right)$ is not true.

4. $\left(\mathrm{L}_{u^{\prime}}\right)$ is true.

$\left(\mathrm{L}_{u}\right)$ is the original liar utterance, uttered at context $u$. As before, after a failed attempt to attach to $\left(\mathrm{L}_{u}\right)$ a semantic value, we infer that it is defective. As it is defective, we reason that the liar utterance is not true. This is $\left(\mathrm{L}_{u^{\prime}}\right)$, an utterance whose sentence is the same as that of the liar, but it is uttered at a different context, $u^{\prime}$. Now this latter utterance seems to have been properly inferred, so it is finally claimed to be true. By contrast to the non-relativized formulation, here there is no explicit contradiction between lines (2) and (4): an utterance involving the same sentence is assessed to be at once defective and at once true, but each time a different context of use is involved.

When attaching subscripts to $(\mathrm{L})$ we had to make a choice as to whether the utterance referred to in line (3) is the liar utterance from line (1). Obviously, it should be. Taking a subscript other than $u$ at (3) would amount to changing the subject, and the claim made at that line would no longer follow. The same goes for line (2). Thus, from line (1) to line (3) we are concerned with the semantic value of one utterance, namely $\left(\mathrm{L}_{u}\right)$ that has been uttered at (1). All of the utterances in (1)-(3) are assessments of $\left(\mathrm{L}_{u}\right)$ by our definitions. The assessments in (2) and (3) were both inferred, and are taken to be true in this reasoning (and the latter is explicitly claimed to be true in line (4)). 
The said assessments give different semantic values to $\left(\mathrm{L}_{u}\right)$ : 'defective' and 'not true'. Moreover, these values are conflicting: $\left(\mathrm{L}_{u}\right)$ was found to be defective since, inter alia, assuming it was not true led to a contradiction. Being defective should be understood here as resisting both values 'true' and 'not true' (see (Simmons, 2015)). Thus, by our definitions, $\left(\mathrm{L}_{u}\right)$ is assessment-sensitive.

Note that on the one hand, 'defective' and 'not true' are conflicting values, and on the other hand, the contextualist infers that $(\mathrm{L})$ is not true from its being defective. One can thus question the inference from (2) to (3). To this the contextualist might reply that it is the context shift that makes this inference possible: 'defective' is in conflict with 'not true' when the latter is uttered in the initial context (denying that (L) is 'not true' was part of the reasoning that lead to the conclusion that it is defective), but is consonant with 'not true' as uttered in a later context. One can then press and ask what warrants the inference from (2) to (3). ${ }^{13}$ Sticking to the main issue at hand, I shall give the contextualists this move without attempting to defend it, and see what it implies.

Now, having relativized truth to context of use and revising the liar sentence accordingly, we are led to assessment sensitivity: the same utterance receives conflicting values by true assessments in different contexts-not of use (there is only one context of use for a given utterance)—but rather of assessment.

Note that we have not refuted here the contextualist stance, but merely pointed to an implication of it. A sentence or an expression can be sensitive to both contexts of use and context of assessment (that is the reason the relativist takes both into account for the truth bearers). However, we see that merely a shift of context of use does not fully explain the liar discourse.

Note further, that we are here not endorsing or proposing a new relativistic approach about truth. For a recent defense of a relativistic solution to the liar see (Scharp, 2013). ${ }^{14}$ Moreover, were one to resist identifying the liar discourse as assessments of the semantic theory, we still remain with a form of relativism: we have an utterance receiving two different truth values on two occasions-it is just that now we cannot point to the context of assessments as the additional parameter by which semantic value can be determined.

\footnotetext{
${ }^{13}$ For a criticism along these lines see (Gauker, 2006).

${ }^{14}$ Scharp's arguments rely on the idea that truth is an inconsistent concept, and there is no overlap with the arguments presented here.
} 


\section{From Relativism to Contextualism}

Before we turn to specific contextualists, I would like to briefly note two more entailments between contextualism and relativism related to truth. First, note that assessment sensitivity about any sentence leads to contextualism about truth or some other semantic notion. Let $s$ be an assessment-sensitive sentence, so that there is an utterance $\alpha$ of $s$ and true assessments $\beta$ and $\gamma$ used in contexts $c_{1}$ and $c_{2}$ respectively, such that $\operatorname{val}(\alpha, \beta)$ and $\operatorname{val}(\alpha, \gamma)$ are conflicting. Assume that the sentence of the assessment $\beta, s_{\beta}$, is " $\alpha$ is true". Since $\gamma$ is true in $c_{2}$ and is in conflict with $\beta$, presumably $s_{\beta}$ would be false in $c_{2}$. Thus $s_{\beta}$ 's evaluation depends on the context of its use, and thus we have contextualism about 'true'. Substitute any other semantic predicate for 'true' in $\beta$, and we get contextualism with respect to that predicate. In general: assessment sensitivity of a sentence amounts to the dependence of assessments on context of use, and by that of the semantic notions involved in the particular assessments.

Further, MacFarlane points out that relativism about any expression entails relativism about truth:

Note that, if the language can express any assessment-sensitive propositions, "true" will also be assessment-sensitive, since if $p$ is assessmentsensitive, the proposition that $p$ is true must be assessment-sensitive too. (MacFarlane, 2014, 93)

In MacFarlane's setting, it is the propositions that are said to be assessment-sensitive (so the truth value of a proposition is determined only given a context of assessment). Transforming the quote from MacFarlane to a setting not committed to propositions, we assume that there is some assessment-sensitive sentence $s$ in the language. Thus, there is an utterance $\alpha$ of $s$ that is assessment-sensitive. Let $c_{1}$ and $c_{2}$ be two contexts of assessment on which $\alpha$ receives conflicting values, and let $\beta$ and $\gamma$ be the true assessments expressing those values respectively. Let $\hat{s}$ be the sentence "s is true", and $\hat{\alpha}$ an utterance of $\hat{s}$ in the same context of use as that of $\alpha$. Let $\hat{\beta}$ and $\hat{\gamma}$ be assessments expressed at $c_{1}$ and $c_{2}$ assigning $\operatorname{val}(\alpha, \beta)$ and $\operatorname{val}(\alpha, \gamma)$ to $\hat{\alpha}$ respectively. By the transparency of truth (relativised to context-that much is assumed by the contextualists we discuss), the truth value of $s$ and the truth value of $\hat{s}$ should be equal in every pair of context of use and context of assessment, and thus $\hat{\beta}$ and $\hat{\gamma}$ are true assessments of $\hat{\alpha}$ assigning to it conflicting values. 


\section{Two Case Studies}

\subsection{Simmons}

To make my critical claim of contextualism more concrete, I propose to examine two instances of the brand of contextualism that I delineated in broad strokes. Both Simmons and Glanzberg appeal to a version of the strengthened liar discourse and handle it by relativizing to contexts of use. Simmons (2015) has a version of the strengthened liar discourse that is very similar to the one presented in $\S 2$. In his scenario, we encounter a sentence written on the board saying "The sentence on the board is not true”. We label this sentence token by '(L)'. After some deliberation, we conclude that (L) is defective, and thus produce the sentence token (R): "The sentence on the board is not true". (R), according to Simmons, is a genuine repetition of $(\mathrm{L})$ : "both sentences $(\mathrm{L})$ and $(\mathrm{R})$ are comprised of the same words with the same meanings and extensions" (Simmons, 2015, §2). Yet according to our reasoning, (L) is defective, while (R) is true.

Here enters the role of the context shift: (L) and (R) occur at different contexts: an unreflective context and a reflective context respectively. Note that here, for Simmons, $(\mathrm{L})$ and $(\mathrm{R})$ are already relativized to contexts of use. Each context of use comes with an appropriate truth predicate and a Tarskian truth-schema. Relativizing truth to

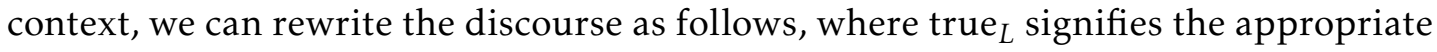
truth predicate for the context of $(\mathrm{L})$, and and true $\mathrm{r}_{r L}$ signifies the appropriate truth predicate for the reflective context of $(\mathrm{R})$ :

1. (L): The sentence on the board is not true ${ }_{L}$.

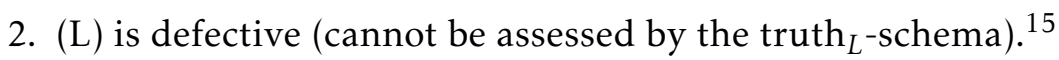

3. (R): The sentence on the board is not true ${ }_{L}$.

4. (R) is true ${ }_{r L}$ (as assessed by the truth ${ }_{r L}$-schema). ${ }^{16}$

Note that 'true' in both (L) and (R) (lines (1) and (3)) is assigned the same subscript: that is so since all the stages from (1) to (3) are concerned with whether the sentence on the board is true ${ }_{L}$. However, the truth-schemas used for assessing (L) in line (1) and $(\mathrm{R})$ in line (3) are different, and set by the context. The truth ${ }_{L}$-schema is the one

\footnotetext{
${ }^{15}$ The instance for $(\mathrm{L})$ in the $\operatorname{truth}_{L}$-schema is: $(\mathrm{L})$ is $\operatorname{true}_{L}$ iff $(\mathrm{L})$ is not true . .

${ }^{16}$ The instance for $(\mathrm{R})$ in the $\operatorname{truth}_{r L}$-schema is: $(\mathrm{R})$ is true ${ }_{r L}$ iff $(\mathrm{L})$ is not true . .
} 
appropriate for the context of (L), and when employed, (L) is found to be defective. The truth $r L^{-s c h e m a}$ is the one appropriate for the context of $(\mathrm{R})$, and it rules $(\mathrm{R})$ to be true (as expressed in line (4)).

The way the extensions of the truth predicate in context are determined is set by Simmons in his Singularity Theory. Simmons notes that his contextual approach is also compatible with a Tarskian hierarchy of extensions for the truth predicate. But by contrast, Simmons's Singularity Theory only minimally restricts any particular use of 'true', so that rather than being relegated just one level in the Tarskian hierarchy, it would be generally applicable-except for certain singularities (pathologies), where the application breaks down (Simmons, 2015, §3).

I shall not go into the details of Simmons's Singularity Theory, as it is the contextual basis that is here at stake. On that matter, we note that Simmons lays a heavy weight on the phenomenon of repetition. The fact that the same sentence is evaluated differently on different occasions of use is used by Simmons as a primary motivation for the relativization to context. ${ }^{17}$

Nevertheless, the weight put on repetition is unwarranted. The point of line (3) is to assess (L). Remember that in the current story (L) is a sentence token. The fact that we use the same sentence as that of $(\mathrm{L})$ to express this assessment is coincidental. We could have used a different sentence to express the same content, and remain faithful to the gist of the discourse. For instance, instead of referring to $(\mathrm{L})$ as 'the sentence on the board', we could have used 'the sentence written at 16:35', etc., or just '(L)'. In such an alternative scenario, the sentence of (L) would not have been repeated. Nonetheless, the reasoning would have gone through. In line (4) we would still ascribe truth to the assessment made in line (3).

Now in the alternative scenario, as well as in the original one, the same sentence token $(\mathrm{L})$ is assessed in conflicting ways at different stages, exactly like in the description we gave in the beginning of the section. This, we have shown, entails relativism in the broad sense, and given the assumptions that we made, also assessment sensitivity: Simmons is thus clearly, even if not explicitly, committed to a form of semantic relativism. The alternative scenario shows us that the focus on repetition distracts us from important features of the liar discourse: it is not essential to the gist of the

\footnotetext{
${ }^{17}$ In previous work (Simmons, 1993), Simmons does not use the phenomenon of repetition as a primary motivation for his contextualist approach. However, the essence of the contextualist approach is the same, as is the implication to relativism.
} 
discourse, and when the liar is not repeated we can no longer resort to a shift in its context of use as an explanation for the conflicting evaluations.

Simmons further adds that $(\mathrm{L})$ can be rehabilitated: it can be assessed by the reflective truth ${ }_{r L}$-schema, so that while it is not true ${ }_{L},(\mathrm{~L})$ is true $r_{L}$. This point of Simmons is a further (implicit) concession to relativism: the same utterance, assessed before as defective and later as not true, is also assessed as true-using both the truth predicate and truth schema relativized to the reflective context.

\subsection{Glanzberg}

Glanzberg too describes a form of strengthened liar reasoning. Unlike Simmons, who works with sentence types in context, Glanzberg takes propositions as truth bearers, and formulates the liar as saying that it does not express a true proposition. We shall follow his presentation using a formalized language with specified principles.

Glanzberg (2001) starts out by presenting the liar discourse un-relativized to context. He introduces the following notation:

Let $s$ be a sentence and $p$ a proposition. We use $\operatorname{Exp}(s, p)$ to signify that $s$ expresses $p$, and $T(p)$ that $p$ is true. The liar sentence is formalized as (we suppress quotation marks henceforth for ease of notation):

$$
l: \neg \exists p(\operatorname{Exp}(\ulcorner l\urcorner, p) \wedge T(p))
$$

Glanzberg employs the following principles, all reasonable given the interpretation of Exp and T:

$(U-\operatorname{Exp}) \quad(\operatorname{Exp}(s, p) \wedge \operatorname{Exp}(s, q)) \rightarrow p=q$

$(T-I d) \quad p=q \rightarrow(T p \leftrightarrow T q)$

$(T-\operatorname{Prop}) \quad \operatorname{Exp}(s, p) \rightarrow(T p \leftrightarrow \Sigma)$ for ' $\Sigma$ ' a sentence and 's'

a sentence name for ' $\Sigma$ '.

(Exp-Prov) If $s$ is provable from true premises, then

$\exists p(\operatorname{Exp}(s, p))$. 
Now when carrying out the liar discourse, we start out by assuming that $l$ expresses a proposition, and reaching a contradiction:

1. Assume $\operatorname{Exp}(l, q)$.

2. Now suppose $T q$.

3. $\neg \exists p(\operatorname{Exp}(l, p) \wedge T p) \quad($ by $(T-\operatorname{Prop}))$

4. $\neg T q \quad$ (from (1) and the assumption that $\operatorname{Exp}(l, q)$ )

5. Suppose $\neg T q$.

6. $\exists p(\operatorname{Exp}(l, p) \wedge T(p)) \quad($ by $(T-\operatorname{Prop}))$

7. $p=q \quad$ (from $(6),(U-\operatorname{Exp})$ and $\operatorname{Exp}(l, q))$

8. $T q \quad$ (from (6), $(7)$ and $(T-I d))$

Supposing either $T q$ or $\neg T q$ leads to contradiction, so we have:

9. $\neg \exists p(\operatorname{Exp}(l, p))$

But then:

10. $\neg \exists p(\operatorname{Exp}(l, p) \wedge T p)$

Which is just:

11. $l$

So after all:

12. $\exists p(\operatorname{Exp}(l, p)) \quad$ (from $(\operatorname{Exp}-$ Prov $))$

(See (Glanzberg, 2001, 229))

We see that, as before, $l$ in its first instance is defective (fails in trying to say that it itself does not express a true proposition), but is then inferred later in the course of reasoning. Glanzberg then argues that there is a context shift along the way. 
For considerations of space, the technical details of Glanzberg's proposal cannot be presented here (for the details, consult (Glanzberg, 2001, 2004)). However, it would be useful to go into just sufficient detail to show the implications of Glanzberg's view, and in particular to show that what he calls "extraordinary context sensitivity" is indeed a type of assessment sensitivity.

In broad lines, Glanzberg's proposal involves an expansion of the domain of propositions. In the initial context, $l$ does not express a proposition-there is no proposition for $l$ to express. By the end of the reasoning, the domain of propositions expands, i.e., more truth conditions become accessible (Glanzberg, 2001, 241), (Glanzberg, 2004, 39). At the later context, there is a proposition for $l$ to express. However, note a subtle, yet crucial feature: it cannot be that $l$, as uttered in the later context has a proposition to express. This would lead to contradiction, exactly as it would in the initial context. It is $l$, as uttered in the initial context, that finally obtains a proposition to express. This leads us to relativism, at least in the broad sense: the same utterance (i.e. sentence in context) receives conflicting semantic evaluations. At first it does not express a proposition, and then it expresses a true one.

In the earlier paper (2001) Glanzberg proposes the idea of a context shift, and that the domain of propositions expands. In (2004) Glanzberg provides an elaborate formal apparatus to show how this works. Let us then focus on the presentation in the latter, more developed paper.

To model the context shift, Glanzberg employs definability theory and admissible sets. The domain of propositions available at a context is determined by the truth conditions available in a context, which in turn are determined by a salience structure exhibiting the semantic relations available, by reflecting salient domains, individuals, and relations at a given point in a discourse $(2004$, p. 39). The domain of propositions is given by a certain admissible sets construction applied to the salience structure provided by context. Each addition of a semantic relation expands the structure. We obtain a hierarchy of structures: each building on the previous one with added expressive power.

The context sensitivity of the liar, as explained in (2004), is not due to an indexical or any ordinary context-sensitive component: it is the domain of the quantifier that shifts through contexts. Therefore, the formulation of the liar sentence remains as is (for instance, the Exp relation is not parameterised to contexts as Glanzberg initially 
suggested in (2001)). Glanzberg explains that the liar sentence exhibits extraordinary context dependence, as we do not ordinarily presume that the quantifier is restricted when ranging over propositions (2004, p. 33).

The extraordinariness of the liar sentence does not lie merely in the expansion of the domain of propositions. Let us start with a simplistic description of the liar reasoning and refine it until we get a better handle of what is going on. We have two utterances of the liar sentence with different semantic values. The (extraordinary) context dependence of the liar and the context shift at work explain this, and so we have a contextual solution. So far so good. Now, the first instance of the liar says of itself that it does not express a true proposition. It thus leads to paradox, unless we ban it from expressing a proposition. The second instance of the liar does not say of itself that it does not express a true proposition: had it done so we'd be stuck in paradox. The second instance thus says that the first does not express a true proposition-in the original context.

We are close to announcing assessment sensitivity, but not quite there yet. The two instances of the liar have different semantic values, though they seem to say (or attempt to say) the same thing. From this one might relate Glanzberg's extraordinary context dependence to the now in use term nonindexical contextualism, by which the same content may receive different semantic values as expressed in different contexts. $^{18}$

Some have referred to the view of nonindexical contextualism as a kind of relativism about truth. This is not my intention: my claim is stronger. Nonindexical contextualism is still short of relativism as I have used the term in that by nonindexical contextualism the context of use suffices for providing values to all parameters relevant for semantic value, while here, I would like to claim, parameters from the context of assessment come into play. Indeed, it seems to me that Glanzberg's extraordinary contextualism is a proto-relativism. ${ }^{19}$ To see this, we need, as we did before, to divert our focus from the fact that the liar sentence is uttered twice in the reasoning to the fact that the liar sentence is assessed twice. Accepting the statements in the

\footnotetext{
${ }^{18}$ The parameters leading to differing semantic values might not be the usual ones of worlds and times that are in play in "ordinary" indexical contextualism, but rather coordinates as tastes and aesthetic standards.

${ }^{19}$ I use the term "proto-relativism" to flag that Glanzberg's work appeared at an incipient stage of the current debate with its current terminology.
} 
discourse as bona fide assessments, we shall also obtain assessment sensitivity. ${ }^{20}$ We thus focus on lines 9 and 11 above. Glanzberg describes the situation in the earlier paper:

...we have found a sentence such that an utterance of it at one moment appears unable to say anything at all, but then appears to say something true. Indeed, we first prove that it cannot express a proposition, and then subsequently prove that it can. (Glanzberg, 2001, 230)

I claim: "it" in Glanzberg's quote refers (or ought to refer) to the initial utterance of the liar sentence-it is the same utterance (or content thereof) that is given differing semantic values. Let (A) be the context of line 9: $\neg \exists p(\operatorname{Exp}(l, p))$ and (B) be the context of line 11: l. Indeed, Glanzberg writes:

...the trivial step is one which would roughly be reported as 'As used in context (A), $l$ does not express a true proposition'. We thus find ourselves needing to make reference to the semantics potentials of sentences in context, in order to clearly state the logically trivial inference. (2004, p. 68 , my emphasis)

While a merely contextualist approach, indexical or nonindexical, will have us look at one context for assigning semantic value, in Glanzberg's description, in the latter context in the liar reasoning we consider two contexts at once:

...speakers need to identify features of the prior context, and on the basis of them work out how to express aspects of its semantics from their new context. (2004, p. 69)

Speakers do this since it is the content of the claims made in the initial context that are evaluated (p. 74), and they therefore hold fixed the semantic relations denoted by the existential quantifier and the truth predicate as they were interpreted in the initial context (p. 75), thus:

The conclusion at (B) is made by way of an internal truth relation, which reconstructs the semantics of the prior context (A). This is a kind of reflection on the semantic potentials of sentences in context (A). The proposition

\footnotetext{
${ }^{20}$ Note, however, that the type of relativism attributed to Glanzberg here is content relativism. That is, propositions here remain non-relative, but which proposition (or whether a proposition) is expressed by a sentence is relative to both context of use and context of assessment.
} 
expressed at (B) bears this out, as it winds up saying that the Liar sentence cannot express a true proposition in the (A) context. (2004, p. 81)

Viewing Glanzberg's proposal in the current light, we now consider (A) and (B) as contexts of assessment of the liar sentence as uttered in (A) (henceforth: the liar utterance). According to Glanzberg's own view, context (A) lacks the resources to assess the liar utterance. The assessment fails, and as in previous sections, we may deem the liar utterance as assessed from (A) to be defective (as is its assessment-as it is its own assessment). In context (B) we obtain the relevant resources to evaluate the liar utterance and conclude that it does not express a true proposition. Beyond nonindexical contextualism, by which the same content is expressed twice and receives different values, here the same content (fixed by the initial context) is assessed twice and receives different values: 'defective' and 'does not express a true proposition' (which are conflicting by the same explanation given in previous sections). And the crucial difference lies in the resources available at each context for making an assessment, while the context of use of the assessed sentence remains fixed. Admittedly, our explanation relies on an unconventional stock of semantic values ('defective' and 'does not express a true proposition'), but the point really is that what comes out of an assessment depends on the context in which it is made, over and above the context in which the assessed content was expressed. Glanzberg's elaborate apparatus for modelling the interplay of the context of use in (A) and context of assessment in (B) is probably the most sophisticated account of assessment sensitivity to date.

To conclude: Glanzberg presents his theory as contextualism regarding contexts of use (even if an extraordinary one). Indeed, we have a sentence, namely $l$, that is uttered twice and receives different evaluations. However, I would like to stress that as in the Simmons case, repetition of $l$ is immaterial. We could reformulate the discourse in a faithful manner where $l$ is not repeated. The gist of the discourse is that $l$, as uttered in the initial context, is reassessed, so the relevant context shift concerns assessments. Thus, both Simmons and Glanzberg are in fact relativists since they have a sentence uttered in one given context that receives conflicting semantic evaluations.

\section{Conclusion}

Contextualists about truth focus on the use of the liar sentence in the liar discourse. However, the liar discourse is primarily concerned with assessing the liar. As pointed 
out, we might conceive of an alternative discourse with the same concern, where the liar is used only once. On the other hand, it is essential that the liar be mentioned more than once, as it is the same item we are interested in evaluating throughout: it is the conflicting evaluations that lead into trouble. Thus, if we attribute the difference in evaluations at different stages to a shift in context, it seems that a relativist explanation is more appropriate. Moreover, the contextualists' appeal to the reflective or unreflective status of contexts further enforces taking the assessor's perspective: reflection is part and parcel of the process of semantic evaluation. And even if one were to reject the appeal to assessments in the object language, we still find here an implication of relativism in the broad sense, as contexts of use are still insufficient to determine semantic value.

This is not a defense of a relativistic solution to the liar. My purpose here was simply to observe a connection between contextualism and relativism. Consequently, when discussing Simmons and Glanzberg my only criticism was on the partial presentation of their theory.

Now that we have seen that the contextualist line of thought leads to relativism, we may ask whether, from this new perspective, we have a viable theory with respect to the liar. Initially, it seems that looking at the stages of the liar discourse as assessments captures an important feature of that discourse. It remains to be seen if the emerging relativism is worth pursuing. ${ }^{21}$

${ }^{21}$ The relativist approach that emerges has a curious feature that should be taken into consideration. The liar discourse becomes essentially assessment-sensitive. Observe the following reformulation of the strengthened liar discourse, taking into account that truth is relative to context of assessment (we only need to be concerned with the first three steps):

1. $\left(\mathrm{L}_{u}^{a}\right):\left(\mathrm{L}_{u}^{a}\right)$ is not true.

2. $\left(\mathrm{L}_{\mathfrak{u}}^{a}\right)$ is defective.

3. $\left(\mathrm{L}_{u}^{a}\right)$ is not true.

The sentence in the first line might be more easily read as: "The sentence $\mathrm{L}$, as uttered in context $u$ and assessed from context $a$ is not true." By similar reasoning to what we had before, we can move from (1) to (2) to (3). So we end up having two conflicting assessments of the very same item, which is already relativized to a context of assessment. And applying the contextualist reasoning, we solve the problem by reference to a context shift throughout the stages of the discourse. Context sensitivity will resurface no matter how many contextual parameters are fixed explicitly in the discourse-there is no way to reformulate the reasoning in the semantic theory in such a way that the sentences involved obtain an absolute truth value. One might object to the explicit reference to context of assessment in the discourse 


\section{Acknowledgements}

This paper originated from comments to Keith Simmons given at the Joint Workshop of The Hebrew University and The University of North Carolina at Chapel Hill in 2013. It was then presented at the Third Workshop on Philosophy of Logic of the Munich - Buenos Aires Logic Group and at the Philosophy Department at the University of Konstanz. I thank the audiences there for useful comments. I also wish to thank the following for very helpful comments and discussion on earlier drafts: Ole Hjortland, Dirk Kindermann, Hannes Leitgeb, Julien Murzi, Stewart Shapiro, Keith Simmons, Paula Teijeiro and two anonymous referees for this journal. This work has benefited from the support of the Alexander von Humboldt Foundation.

\section{References}

Barwise, J., \& Etchemendy, J. (1987). The liar: An essay on truth and circularity. Oxford: Oxford University Press.

Burge, T. (1979). Semantical paradox. The Journal of Philosophy, 76(4), 169-198.

Cappelen, H. (2008). Content relativism and semantic blindness. Relative truth, 26586.

Egan, A., Hawthorne, J., \& Weatherson, B. (2005). Epistemic modals in context. In G. Preyer \& G. Peter (Eds.), Contextualism in philosophy (pp. 131-168). Oxford: Oxford University Press.

García-Carpintero, M., \& Kölbel, M. (Eds.). (2008). Relative truth. Oxford University Press.

Gauker, C. (2006). Against stepping back: A critique of contextualist approaches to the semantic paradoxes. Journal of Philosophical Logic, 35(4), 393-422.

Glanzberg, M. (2001). The liar in context. Philosophical Studies, 103(3), 217-251.

Glanzberg, M. (2004). A contextual-hierarchical approach to truth and the liar paradox. Journal of Philosophical Logic, 33(1), 27-88.

Kaplan, D. (1989). Demonstratives: An essay on the semantics, logic, metaphysics, and epistemology of demonstratives and other indexicals. In Themes from Kaplan (p. 481-566).

(which would mean a serious limitation of expression in the semantic theory!)—but then, a fortiori, the liar discourse remains assessment-sensitive. 
Lewis, D. (1979). Scorekeeping in a language game. Journal of Philosophical Logic, $8(1), 339-359$.

MacFarlane, J. (2005). Making sense of relative truth. In Proceedings of the aristotelian society (hardback) (Vol. 105, pp. 305-323).

MacFarlane, J. (2014). Assessment sensitivity: Relative truth and its applications. Oxford: Calderon Press.

Parsons, C. (1974). The liar paradox. Journal of Philosophical Logic, 3(4), 381-412.

Scharp, K. (2013). Truth, the liar, and relativism. Philosophical Review, 122(3), 427510.

Simmons, K. (1993). Universality and the liar: An essay on truth and the diagonal argument. Cambridge University Press.

Simmons, K. (2015). Paradox, repetition, revenge. Topoi, 34(1), 121-131.

Stalnaker, R. (1978). Assertion. In P. Cole (Ed.), Syntax and semantics, volume 9 (p. 315-332). New York: Academic Press. 